Bulletin of the Section of Logic

Volume 48/3 (2019), pp. 173-185

http://dx.doi.org/10.18778/0138-0680.48.3.02

Young Bae Jun and Xiao Long Xin*

\title{
COMPLEX FUZZY SETS WITH APPLICATION IN BCK/BCI-ALGEBRAS
}

\begin{abstract}
As a generation of fuzzy set, the notion of complex fuzzy set which is an innovative concept is introduced by Ramot, Milo, Friedman and Kandel. The purpose of this article is to apply complex fuzzy set to BCK/BCI-algebras. The notions of a complex subalgebra and a complex left (right) reduced ideal in a BCK/BCIalgebra are introduced, and related properties are investigated. Characterizations of a complex subalgebra are provided, and the homomorphic image (preimage) of a complex subalgebra and a complex left (right) reduced ideal.
\end{abstract}

Keywords: complex $t$-norm, min-complex $t$-norm, complex subalgebra, complex left (right) reduced ideal, complex characteristic function.

Mathematics Subject Classification (2010): 06F35, 03E72, 08A72.

\section{Introduction}

The extension of crisp sets to fuzzy sets, in terms of membership functions, is mathematically comparable to the extension of the set of integers, $\mathbb{Z}$, to the set of real numbers, $\mathbb{R}$. That is, expanding the range of the membership function, $\mu_{A}(x)$, from $\{0,1\}$ to $[0,1]$ is mathematically analogous to the extension of $\mathbb{Z}$ to $\mathbb{R}$. Another extension of fuzzy set theory, Romat et al. [1] introduced the innovative complex fuzzy set. The complex fuzzy set, $A$, is characterized by a membership function, $\mu_{A}$, whose range is not limited to $[0,1]$ but extended to the unit circle in the complex plane. Hence,

${ }^{*}$ Corresponding author. 
$\mu_{A}(x)$ is a complex valued function that assigns a grade of membership of the form $r_{A} \cdot e^{j \omega_{A}(x)}$, where $j=\sqrt{-1}$, to any element $x$ in the universe of discourse. The value of $\mu_{A}(x)$ is defined by the two variables, $r_{A}(x)$ and $\omega_{A}(x)$, both real-valued, with $r_{A}(x) \in[0,1]$. Tamir and Kandel [2] proposed an axiomatic framework for first order predicate complex fuzzy logic and use this framework for axiomatic definition of complex fuzzy classes. Al-Qudah and Hassan [3] introduced the concept of complex multi-fuzzy sets as a generalization of the concept of multi-fuzzy sets by adding the phase term to the definition of multi-fuzzy sets, and provided the structure of distance measure on complex multi-fuzzy sets by extending the structure of distance measure of complex fuzzy sets.

The aim of this paper is to apply the notion of complex fuzzy sets to $B C K / B C I$-algebras, and to generalize the fuzzy set theory in $B C K / B C I$ algebras. We introduce the notion of a complex subalgebra and a complex reduced left (right) ideal in a BCK/BCI-algebra, and investigate related properties. We provide characterizations of a complex subalgebra. We discuss the homomorphic image (preimage) of a complex subalgebra and a complex left (right) reduced ideal.

\section{Preliminaries}

By a $B C I$-algebra, we mean an algebra $(X, *, 0)$ of type $(2,0)$ satisfying the axioms:

(I) $(\forall x, y, z \in X)(((x * y) *(x * z)) *(z * y)=0)$,

(II) $(\forall x, y \in X)((x *(x * y)) * y=0)$,

(III) $(\forall x \in X)(x * x=0)$,

(IV) $(\forall x, y \in X)(x * y=y * x=0 \Rightarrow x=y)$.

We can define a partial ordering $\leq$ by $x \leq y$ if and only if $x * y=0$. If a BCI-algebra $X$ satisfies $0 * x=0$ for all $x \in X$, then we say that $X$ is a $B C K$-algebra. A nonempty subset $L$ of a $B C K / B C I$-algebra $X$ is called a subalgebra of $X$ if $x * y \in L$ for all $x, y \in L$. We refer the reader to the books $[4,5]$ for further information regarding $B C K / B C I$-algebras.

In 2011, Azam et al. [6] introduced the notion of complex valued metric space which is a generalization of the classical metric space, by defining the partial order " $\preceq$ " on the set of complex numbers.

Let $\mathbb{C}$ be the set of complex numbers and $z_{1}, z_{2} \in \mathbb{C}$. Define a partial order " $\preceq$ " on $\mathbb{C}$ as follows: 


$$
z_{1} \preceq z_{2} \text { if and only if } \operatorname{Re}\left(z_{1}\right) \leq \operatorname{Re}\left(z_{2}\right) \text { and } \operatorname{Im}\left(z_{1}\right) \leq \operatorname{Im}\left(z_{2}\right),
$$

that is, $z_{1} \preceq z_{2}$ if one of the following holds

(C1) $\operatorname{Re}\left(z_{1}\right)=\operatorname{Re}\left(z_{2}\right)$ and $\operatorname{Im}\left(z_{1}\right)=\operatorname{Im}\left(z_{2}\right)$,

(C2) $\operatorname{Re}\left(z_{1}\right)<\operatorname{Re}\left(z_{2}\right)$ and $\operatorname{Im}\left(z_{1}\right)=\operatorname{Im}\left(z_{2}\right)$,

(C3) $\operatorname{Re}\left(z_{1}\right)=\operatorname{Re}\left(z_{2}\right)$ and $\operatorname{Im}\left(z_{1}\right)<\operatorname{Im}\left(z_{2}\right)$,

(C4) $\operatorname{Re}\left(z_{1}\right)<\operatorname{Re}\left(z_{2}\right)$ and $\operatorname{Im}\left(z_{1}\right)<\operatorname{Im}\left(z_{2}\right)$.

Ramot et al. [1] introduced the notion of complex fuzzy sets.

A complex fuzzy set $A$, defined on a universe of discourse $X$, is characterized by a membership function $\mu_{A}(x)$ that assigns any element $x \in X$ a complex valued grade of membership in $X$, that is, the complex fuzzy set $A$ may be represented as the set of ordered pairs

$$
A=\left\{\left(x, \mu_{A}(x)\right) \mid x \in X\right\},
$$

where $\mu_{A}(x)=r_{A}(x) \cdot e^{j \omega_{A}(x)}, j=\sqrt{-1}, r_{A}(x)$ and $\omega_{A}(x)$ are both realvalued, and $r_{A}(x) \in[0,1]$. Evidently, each complex grade of membership is defined by an amplitude term $r_{A}(x)$ and a phase term $\omega_{A}(x)$.

\section{Complex subalgebras}

Let $A$ and $B$ be complex fuzzy sets on $X$ with complex valued membership functions $\mu_{A}$ and $\mu_{B}$, respectively. We define

$$
\mu_{A}(y) \odot \mu_{B}(z)=\left[r_{A}(y) \diamond r_{B}(z)\right] \cdot e^{j\left[\omega_{A}(y) \bar{\diamond} \omega_{B}(z)\right]},
$$

for all $y, z \in X$ where $\diamond$ is a $t$-norm and $\bar{\diamond}$ is a function

$$
\bar{\diamond}:[0, \pi] \times[0, \pi] \rightarrow[0, \pi]
$$

satisfying the following conditions.

1. $(a \bar{\diamond} b) \bar{\diamond} c=a \bar{\diamond}(b \bar{\diamond} c)$,

2. $a \bar{\diamond} b=b \bar{\diamond} a$,

3. $b \leq c \Rightarrow a \bar{\diamond} b \leq a \bar{\diamond} c$,

4. $a \bar{\diamond} \pi=a$,

where $a, b$ and $c$ are elements of $[0, \pi]$. We say that the function $\bar{\diamond}$ is an extended t-norm, and the operation $\odot$ is the complext-norm. 
In what follows, let $X$ be a BCK/BCI-algebra and consider a complex fuzzy set $A$ on $X$ with complex valued membership function

$$
(\forall x \in X)\left(\mu_{A}(x)=r_{A}(x) \cdot e^{j \omega_{A}(x)}\right)
$$

where $j=\sqrt{-1}, r_{A}(x) \in[0,1]$ and $\omega_{A}(x) \in[0, \pi]$. It will be denoted by

$$
A=\left\{\left(x, \mu_{A}(x)\right) \mid x \in X\right\} .
$$

For any $\delta=r \cdot e^{j \omega}$ with $r \in[0,1]$ and $\omega \in[0, \pi]$, the $\delta$-level set of $A$ is denoted by $[A]_{\delta}$ and is defined to be the set

$$
[A]_{\delta}:=\left\{x \in X \mid \mu_{A}(x) \succeq \delta\right\} .
$$

If, in the complex $t$-norm $\odot$, both the $t$-norm and extended $t$-norm are considered as "min", it is denoted by $\odot_{\min }$ and is called the min-complex t-norm.

Definition 3.1. A complex fuzzy set $A=\left\{\left(x, \mu_{A}(x)\right) \mid x \in X\right\}$ on $X$ is called a complex subalgebra of $X$ if

$$
\left\{\begin{array}{l}
\operatorname{Re}\left(\mu_{A}(x * y)\right) \geq \operatorname{Re}\left(\mu_{A}(x) \odot \mu_{A}(y)\right), \\
\operatorname{Im}\left(\mu_{A}(x * y)\right) \geq \operatorname{Im}\left(\mu_{A}(x) \odot \mu_{A}(y)\right)
\end{array}\right.
$$

or, equivalently, $\mu_{A}(x * y) \succeq \mu_{A}(x) \odot \mu_{A}(y)$ for all $x, y \in X$.

Note that the condition (3.2) is equivalent to the following condition:

$$
\left\{\begin{array}{l}
r_{A}(x * y) \cdot \cos \omega_{A}(x * y) \geq\left(r_{A}(x) \diamond r_{A}(y)\right) \cdot \cos \left(\omega_{A}(x) \bar{\diamond} \omega_{A}(y)\right), \\
r_{A}(x * y) \cdot \sin \omega_{A}(x * y) \geq\left(r_{A}(x) \diamond r_{A}(y)\right) \cdot \sin \left(\omega_{A}(x) \bar{\diamond} \omega_{A}(y)\right)
\end{array}\right.
$$

EXAmple 3.2. Let $X=\{0, a, b, c\}$ be a $B C K$-algebra with the operation * which is described by Table 1 (see [5]).

Let $A$ be a complex fuzzy set on $X$ with the complex valued membership function $\mu_{A}$ defined by

$$
\mu_{A}(x)= \begin{cases}0.7 e^{j \frac{3 \pi}{8}} & \text { if } x=0 \\ 0.5 e^{j \frac{3 \pi}{8}} & \text { if } x=a \\ 0.3 e^{j \frac{3 \pi}{8}} & \text { if } x=b \\ 0.1 e^{j \frac{3 \pi}{8}} & \text { if } x=c .\end{cases}
$$


Table 1. Cayley table of the operation $*$

\begin{tabular}{lllll}
\hline$*$ & 0 & $a$ & $b$ & $c$ \\
\hline 0 & 0 & 0 & 0 & 0 \\
$a$ & $a$ & 0 & 0 & $a$ \\
$b$ & $b$ & $b$ & 0 & $b$ \\
$c$ & $c$ & $c$ & $c$ & 0 \\
\hline
\end{tabular}

It is routine to verify that $A=\left\{\left(x, \mu_{A}(x)\right) \mid x \in X\right\}$ is a complex subalgebra of $X$.

ExAmple 3.3. Let $X=\{0,1, a, b, c\}$ be a $B C I$-algebra in which the operation $*$ is described by Table 2 (see [5]).

Table 2. Cayley table of the operation $*$

\begin{tabular}{llllll}
\hline$*$ & 0 & 1 & $a$ & $b$ & $c$ \\
\hline 0 & 0 & 0 & $a$ & $b$ & $c$ \\
1 & 1 & 0 & $a$ & $b$ & $c$ \\
$a$ & $a$ & $a$ & 0 & $c$ & $b$ \\
$b$ & $b$ & $b$ & $c$ & 0 & $a$ \\
$c$ & $c$ & $c$ & $b$ & $a$ & 0 \\
\hline
\end{tabular}

Let $A$ be a complex fuzzy set on $X$ with the complex valued membership function $\mu_{A}$ defined by

$$
\mu_{A}(x)= \begin{cases}0.7 e^{j \frac{\pi}{8}} & \text { if } x=0, \\ 0.7 e^{j \frac{3 \pi}{16}} & \text { if } x=1, \\ 0.7 e^{j \frac{\pi}{4}} & \text { if } x=b, \\ 0.7 e^{j \frac{5 \pi}{16}} & \text { if } x \in\{a, c\} .\end{cases}
$$

If we use the lexicographical order, denoted by $\prec_{l}$, on $\mathbb{C}$, that is, for any two complex numbers $z_{1}=a_{1}+j b_{1}$ and $z_{2}=a_{2}+j b_{2}$,

$z_{1} \prec_{l} z_{2}$ provided either $a_{1}<a_{2}$ or $a_{1}=a_{2}$ and $b_{1}<b_{2}$, then $A=\left\{\left(x, \mu_{A}(x)\right) \mid x \in X\right\}$ is a complex subalgebra of $X$. 
Proposition 3.4. Let $A=\left\{\left(x, \mu_{A}(x)\right) \mid x \in X\right\}$ be a complex subalgebra of a $B C K$-algebra $X$. If we use the min-complex $t$-norm, then $\mu_{A}(0) \succeq \mu_{A}(x)$ for all $x \in X$.

Proof: Let $x \in X$. Using the conditions (III) and (3.3), we have

$$
\begin{aligned}
\operatorname{Re}\left(\mu_{A}(0)\right) & =r_{A}(0) \cdot \cos \omega_{A}(0)=r_{A}(x * x) \cdot \cos \omega_{A}(x * x) \\
& \geq\left(r_{A}(x) \diamond r_{A}(x)\right) \cdot \cos \left(\omega_{A}(x) \triangleright \omega_{A}(x)\right) \\
& =r_{A}(x) \cdot \cos \omega_{A}(x)=\operatorname{Re}\left(\mu_{A}(x)\right)
\end{aligned}
$$

and

$$
\begin{aligned}
\operatorname{Im}\left(\mu_{A}(0)\right) & =r_{A}(0) \cdot \sin \omega_{A}(0)=r_{A}(x * x) \cdot \sin \omega_{A}(x * x) \\
& \geq\left(r_{A}(x) \diamond r_{A}(x)\right) \cdot \sin \left(\omega_{A}(x) \bar{\diamond} \omega_{A}(x)\right) \\
& =r_{A}(x) \cdot \sin \omega_{A}(x)=\operatorname{Im}\left(\mu_{A}(x)\right) .
\end{aligned}
$$

Therefore $\mu_{A}(0) \succeq \mu_{A}(x)$ for all $x \in X$.

Proposition 3.5. Let $A=\left\{\left(x, \mu_{A}(x)\right) \mid x \in X\right\}$ be a complex subalgebra of a $B C K$-algebra $X$ in which $\mu_{A}$ is increasing. If we use the min-complex t-norm, then $\mu_{A}$ is constant.

Proof: Straightforward.

Theorem 3.6. Let $A=\left\{\left(x, \mu_{A}(x)\right) \mid x \in X\right\}$ be a complex subalgebra of $X$ in which

$$
\mu_{A}(x)=r_{A}(x) \cdot e^{j \omega_{A}(x)}
$$

with $j=\sqrt{-1}, r_{A}(x) \in[0,1]$ and $\omega_{A}(x) \in\left[\frac{\pi}{2}, \pi\right]$. If we use the min-complex $t$-norm, then the $\delta$-level set $[A]_{\delta}$ of $A$ is a subalgebra of $X$ for all $\delta:=r \cdot e^{j \omega}$ with $r \in[0,1], \omega \in\left[\frac{\pi}{2}, \pi\right]$ and $[A]_{\delta} \neq \emptyset$.

Proof: Assume that $A=\left\{\left(x, \mu_{A}(x)\right) \mid x \in X\right\}$ is a complex subalgebra of $X$. Let $x, y \in[A]_{\delta}$. Then $\mu_{A}(x) \succeq \delta$ and $\mu_{A}(y) \succeq \delta$. Thus

$$
\begin{aligned}
& \operatorname{Re}\left(\mu_{A}(x)\right)=r_{A}(x) \cdot \cos \omega_{A}(x) \geq r \cdot \cos \omega, \\
& \operatorname{Im}\left(\mu_{A}(x)\right)=r_{A}(x) \cdot \sin \omega_{A}(x) \geq r \cdot \sin \omega, \\
& \operatorname{Re}\left(\mu_{A}(y)\right)=r_{A}(y) \cdot \cos \omega_{A}(y) \geq r \cdot \cos \omega, \\
& \operatorname{Im}\left(\mu_{A}(y)\right)=r_{A}(y) \cdot \sin \omega_{A}(y) \geq r \cdot \sin \omega .
\end{aligned}
$$

Now, we consider the following four cases.

(1) $r_{A}(x) \geq r_{A}(y)$ and $\omega_{A}(x) \geq \omega_{A}(y)$,

(2) $r_{A}(x) \geq r_{A}(y)$ and $\omega_{A}(x)<\omega_{A}(y)$,

(3) $r_{A}(x)<r_{A}(y)$ and $\omega_{A}(x) \geq \omega_{A}(y)$,

(4) $r_{A}(x)<r_{A}(y)$ and $\omega_{A}(x)<\omega_{A}(y)$. 
The case (1) implies that

$$
\left(r_{A}(x) \diamond r_{A}(y)\right) \cdot \cos \left(\omega_{A}(x) \bar{\diamond} \omega_{A}(y)\right)=r_{A}(y) \cdot \cos \omega_{A}(y) \geq r \cdot \cos \omega
$$

and

$$
\left(r_{A}(x) \diamond r_{A}(y)\right) \cdot \sin \left(\omega_{A}(x) \bar{\diamond} \omega_{A}(y)\right)=r_{A}(y) \cdot \sin \omega_{A}(y) \geq r \cdot \sin \omega .
$$

For the case (2), we have

$$
\begin{aligned}
& \left(r_{A}(x) \diamond r_{A}(y)\right) \cdot \cos \left(\omega_{A}(x) \bar{\diamond} \omega_{A}(y)\right)=r_{A}(y) \cdot \cos \omega_{A}(x) \\
& \geq r_{A}(y) \cdot \cos \omega_{A}(y) \geq r \cdot \cos \omega
\end{aligned}
$$

and

$$
\begin{aligned}
& \left(r_{A}(x) \diamond r_{A}(y)\right) \cdot \sin \left(\omega_{A}(x) \bar{\diamond} \omega_{A}(y)\right)=r_{A}(y) \cdot \sin \omega_{A}(x) \\
& \geq r_{A}(y) \cdot \sin \omega_{A}(y) \geq r \cdot \sin \omega
\end{aligned}
$$

since $\cos \omega_{A}(y) \leq \cos \omega_{A}(x)$ and $\sin \omega_{A}(y) \leq \sin \omega_{A}(x)$. The case (3) induces

$$
\begin{aligned}
& \left(r_{A}(x) \diamond r_{A}(y)\right) \cdot \cos \left(\omega_{A}(x) \bar{\diamond} \omega_{A}(y)\right)=r_{A}(x) \cdot \cos \omega_{A}(y) \\
& \geq r_{A}(x) \cdot \cos \omega_{A}(x) \geq r \cdot \cos \omega
\end{aligned}
$$

and

$$
\begin{aligned}
& \left(r_{A}(x) \diamond r_{A}(y)\right) \cdot \sin \left(\omega_{A}(x) \bar{\diamond} \omega_{A}(y)\right)=r_{A}(x) \cdot \sin \omega_{A}(y) \\
& \geq r_{A}(x) \cdot \sin \omega_{A}(x) \geq r \cdot \sin \omega .
\end{aligned}
$$

From the case (4), we have

$$
\left(r_{A}(x) \diamond r_{A}(y)\right) \cdot \cos \left(\omega_{A}(x) \bar{\diamond} \omega_{A}(y)\right)=r_{A}(x) \cdot \cos \omega_{A}(x) \geq r \cdot \cos \omega
$$

and

$$
\left(r_{A}(x) \diamond r_{A}(y)\right) \cdot \sin \left(\omega_{A}(x) \bar{\diamond} \omega_{A}(y)\right)=r_{A}(x) \cdot \sin \omega_{A}(x) \geq r \cdot \sin \omega .
$$

It follows from (3.2) that

$r_{A}(x * y) \cdot \cos \omega_{A}(x * y) \geq\left(r_{A}(x) \diamond r_{A}(y)\right) \cdot \cos \left(\omega_{A}(x) \bar{\diamond} \omega_{A}(y)\right) \geq r \cdot \cos \omega$ and

$r_{A}(x * y) \cdot \sin \omega_{A}(x * y) \geq\left(r_{A}(x) \diamond r_{A}(y)\right) \cdot \sin \left(\omega_{A}(x) \bar{\diamond} \omega_{A}(y)\right) \geq r \cdot \sin \omega$. Hence $\mu_{A}(x * y) \succeq \delta$, and so $x * y \in[A]_{\delta}$. Therefore $[A]_{\delta}$ is a subalgebra of $X$. 
Corollary 3.7. Let $A=\left\{\left(x, \mu_{A}(x)\right) \mid x \in X\right\}$ be a complex subalgebra of a $B C K$-algebra $X$ in which

$$
\mu_{A}(x)=r_{A}(x) \cdot e^{j \omega_{A}(x)}
$$

with $j=\sqrt{-1}, r_{A}(x) \in[0,1]$ and $\omega_{A}(x) \in\left[\frac{\pi}{2}, \pi\right]$. If we use the min-complex t-norm, then the set

$$
[X]:=\left\{x \in X \mid \mu_{A}(x)=\mu_{A}(0)\right\}
$$

is a subalgebra of $X$.

Proof: Since $\mu_{A}(0) \succeq \mu_{A}(x)$ for all $x \in X$ by Proposition 3.4, we have

$$
[A]_{\mu_{A}(0)}=\left\{x \in X \mid \mu_{A}(x) \succeq \mu_{A}(0)\right\}=\left\{x \in X \mid \mu_{A}(x)=\mu_{A}(0)\right\}=[X] .
$$

It follows from Theorem 3.6 that $[X]$ is a subalgebra of $X$.

Theorem 3.8. Let $A=\left\{\left(x, \mu_{A}(x)\right) \mid x \in X\right\}$ be a complex fuzzy set on $X$ such that the $\delta$-level set $[A]_{\delta}$ of $A$ is a subalgebra of $X$ for all $\delta:=r \cdot e^{j \omega}$ with $r \in[0,1], \omega \in[0, \pi]$ and $[A]_{\delta} \neq \emptyset$. If we use the min-complex $t$-norm, then $A=\left\{\left(x, \mu_{A}(x)\right) \mid x \in X\right\}$ is a complex subalgebra of $X$.

Proof: Suppose that the $\delta$-level set $[A]_{\delta}$ of A is a subalgebra of $X$ for all $\delta:=r \cdot e^{j \omega}$ with $r \in[0,1]$ and $\omega \in[0, \pi]$. Assume that there exist $a, b \in X$ such that

$$
\mu_{A}(a * b) \prec \mu_{A}(a) \odot \mu_{A}(b) .
$$

We take $\delta:=r \cdot e^{j \omega}$ with $r \in[0,1]$ and $\omega \in[0, \pi]$ such that

Then $a * b \notin[A]_{\delta}$,

$$
\mu_{A}(a * b) \prec \delta \preceq \mu_{A}(a) \odot \mu_{A}(b) .
$$

$\left(r_{A}(a) \diamond r_{A}(b)\right) \cdot \cos \left(\omega_{A}(a) \bar{\diamond} \omega_{A}(b)\right)=\operatorname{Re}\left(\mu_{A}(a) \odot \mu_{A}(b)\right) \geq \operatorname{Re}(\delta)=r \cdot \cos \omega$ and

$\left(r_{A}(a) \diamond r_{A}(b)\right) \cdot \sin \left(\omega_{A}(a) \bar{\diamond} \omega_{A}(b)\right)=\operatorname{Im}\left(\mu_{A}(a) \odot \mu_{A}(b)\right) \geq \operatorname{Im}(\delta)=r \cdot \sin \omega$.

It follows that

and

$$
r_{A}(a) \cdot \cos \omega_{A}(a) \geq r \cdot \cos \omega, r_{A}(a) \cdot \sin \omega_{A}(a) \geq r \cdot \sin \omega
$$

$$
r_{A}(b) \cdot \cos \omega_{A}(b) \geq r \cdot \cos \omega, \quad r_{A}(b) \cdot \sin \omega_{A}(b) \geq r \cdot \sin \omega .
$$

This shows that $a, b \in[A]_{\delta}$ and this is a contradiction. Therefore $\mu_{A}(x * y) \succeq$ $\mu_{A}(x) \odot \mu_{A}(y)$ for all $x, y \in X$, and $A=\left\{\left(x, \mu_{A}(x)\right) \mid x \in X\right\}$ is a complex subalgebra of $X$. 
Corollary 3.9. For any subset $L$ of $X$, let $A=\left\{\left(x, \mu_{A}(x)\right) \mid x \in X\right\}$ be a complex fuzzy set on a $B C K$-algebra $X$ with the complex valued membership function $\mu_{A}$ defined by

$$
\mu_{A}(x)= \begin{cases}r_{1} \cdot e^{j \omega_{1}} & \text { if } x \in L, \\ r_{2} \cdot e^{j \omega_{2}} & \text { otherwise }\end{cases}
$$

where $r_{1} \cdot e^{j \omega_{1}} \succeq r_{2} \cdot e^{j \omega_{2}}$. If $L$ is a subalgebra of $X$ and we use the mincomplex $t$-norm, then $A=\left\{\left(x, \mu_{A}(x)\right) \mid x \in X\right\}$ is a complex subalgebra of $X$.

THEOREM 3.10. Let $\delta_{1}, \delta_{2}, \cdots, \delta_{n}, \cdots$ be a strictly increasing sequence of complex numbers, where $\delta_{k}=r_{k} \cdot e^{j \omega_{k}}$ with $r_{k} \in[0,1]$ and $\omega_{k} \in\left[\frac{\pi}{2}, \pi\right]$. For a strictly decreasing sequences $L_{1}(=X), L_{2}, \cdots, L_{n}, \cdots$ of subalgebras of $X$, there is a complex subalgebra $A=\left\{\left(x, \mu_{A}(x)\right) \mid x \in X\right\}$ of $X$ in which

$$
\mu_{A}(x)=r_{A}(x) \cdot e^{j \omega_{A}(x)}
$$

with $j=\sqrt{-1}, r_{A}(x) \in[0,1]$ and $\omega_{A}(x) \in\left[\frac{\pi}{2}, \pi\right]$ such that $[A]_{\delta_{n}}=L_{n}$ for $n \in \mathbb{N}$ if we use the min-complex $t$-norm.

Proof: Define a complex fuzzy set $A$ on $X$ with the complex valued membership function $\mu_{A}$ defined by

$$
\mu_{A}(x)= \begin{cases}\delta_{n} & \text { if } x \in L_{n} \backslash L_{n+1}, \\ \lim _{n \rightarrow \infty} \delta_{n} & \text { if } x \in \cap_{n=1}^{\infty} L_{n} .\end{cases}
$$

It is easy to verify that $A=\left\{\left(x, \mu_{A}(x)\right) \mid x \in X\right\}$ is a complex subalgebra of $X$ and $[A]_{\delta_{n}}=L_{n}$ for $n=1,2, \cdots$.

Let $f: X \rightarrow Y$ be a mapping of sets. If $B$ is a complex fuzzy set on $Y$ with the complex valued membership function $\mu_{B}$, then the preimage of $B$ under $f$, denoted by $f^{-1}(B)$, is also a complex fuzzy set on $X$ with the complex valued membership function $\mu_{f^{-1}(B)}$ which is defined by $\mu_{f^{-1}(B)}(x)=\mu_{B}(f(x))$ for all $x \in X$.

THEOREM 3.11. Let $f: X \rightarrow Y$ be a homomorphism from $X$ to a $B C K / B C I$ algebra $Y$. If $B$ is a complex subalgebra of $Y$ with the complex valued membership function $\mu_{B}$, then the homomorphic preimage $f^{-1}(B)$ of $B$ under $f$ is a complex subalgebra of $X$ with the complex valued membership function $\mu_{f^{-1}(B)}$.

Proof: Assume that $B$ is a complex subalgebra of $Y$ with the complex valued membership function $\mu_{B}$. For any $x, y \in X$, we have 


$$
\begin{aligned}
\operatorname{Re}\left(\mu_{f^{-1}(B)}(x * y)\right) & =\operatorname{Re}\left(\mu_{B}(f(x * y))\right)=\operatorname{Re}\left(\mu_{B}(f(x) * f(y))\right) \\
& \geq \operatorname{Re}\left(\mu_{B}(f(x)) \odot \mu_{B}(f(y))\right) \\
& =\operatorname{Re}\left(\mu_{f^{-1}(B)}(x) \odot \mu_{f^{-1}(B)}(y)\right)
\end{aligned}
$$

and

$$
\begin{aligned}
\operatorname{Im}\left(\mu_{f^{-1}(B)}(x * y)\right) & =\operatorname{Im}\left(\mu_{B}(f(x * y))\right)=\operatorname{Im}\left(\mu_{B}(f(x) * f(y))\right) \\
& \geq \operatorname{Im}\left(\mu_{B}(f(x)) \odot \mu_{B}(f(y))\right) \\
& =\operatorname{Im}\left(\mu_{f^{-1}(B)}(x) \odot \mu_{f^{-1}(B)}(y)\right) .
\end{aligned}
$$

Therefore the homomorphic preimage $f^{-1}(B)$ of $B$ under $f$ is a complex subalgebra of $X$ with the complex valued membership function $\mu_{f^{-1}(B)}$.

THEOREM 3.12. Let $f$ be an endomorphism of $X$. If $A$ is a complex subalgebra of $X$ with the complex valued membership function $\mu_{A}$, then the complex fuzzy set $A[f]$ on $X$ with the complex valued membership function $\mu_{A[f]}$ defined by

$$
\mu_{A[f]}(x)=\mu_{A}(f(x))
$$

for all $x \in X$ is a complex subalgebra of $X$.

Proof: Let $A$ be a complex subalgebra of $X$ with the complex valued membership function $\mu_{A}$. For any $x, y \in X$, we get

$$
\begin{aligned}
\operatorname{Re}\left(\mu_{A[f]}(x * y)\right) & =\operatorname{Re}\left(\mu_{A}(f(x * y))\right)=\operatorname{Re}\left(\mu_{A}(f(x) * f(y))\right) \\
& \geq \operatorname{Re}\left(\mu_{A}(f(x)) \odot \mu_{A}(f(y))\right) \\
& =\operatorname{Re}\left(\mu_{A[f]}(x) \odot \mu_{A[f]}(y)\right)
\end{aligned}
$$

and

$$
\begin{aligned}
\operatorname{Im}\left(\mu_{A[f]}(x * y)\right) & =\operatorname{Im}\left(\mu_{A}(f(x * y))\right)=\operatorname{Im}\left(\mu_{A}(f(x) * f(y))\right) \\
& \geq \operatorname{Im}\left(\mu_{A}(f(x)) \odot \mu_{A}(f(y))\right) \\
& =\operatorname{Im}\left(\mu_{A[f]}(x) \odot \mu_{A[f]}(y)\right) .
\end{aligned}
$$

Therefore $\mu_{A[f]}$ is a complex subalgebra of $X$.

Definition 3.13. A complex fuzzy set $A=\left\{\left(x, \mu_{A}(x)\right) \mid x \in X\right\}$ on $X$ is called a complex left reduced ideal of $X$ if

$$
\operatorname{Re}\left(\mu_{A}(x * y)\right) \geq \operatorname{Re}\left(\mu_{A}(y)\right) \text { and } \operatorname{Im}\left(\mu_{A}(x * y)\right) \geq \operatorname{Im}\left(\mu_{A}(y)\right)
$$

for all $x, y \in X$. If $A=\left\{\left(x, \mu_{A}(x)\right) \mid x \in X\right\}$ satisfies the condition 


$$
\operatorname{Re}\left(\mu_{A}(x * y)\right) \geq \operatorname{Re}\left(\mu_{A}(x)\right) \text { and } \operatorname{Im}\left(\mu_{A}(x * y)\right) \geq \operatorname{Im}\left(\mu_{A}(x)\right)
$$

for all $x, y \in X$, then we say $A=\left\{\left(x, \mu_{A}(x)\right) \mid x \in X\right\}$ is a complex right reduced ideal of $X$.

TheOREM 3.14. Let $A=\left\{\left(x, \mu_{A}(x)\right) \mid x \in X\right\}$ be a complex subalgebra of a $B C K$-algebra $X$. If we use the min-complex t-norm, then $A$ is a complex left reduced ideal of $X$ if and only if the complex valued membership function $\mu_{A}$ of $A$ is constant, that is, $\mu_{A}(0)=\mu_{A}(x)$ for all $x \in X$.

Proof: The sufficiency is clear. Assume that $A$ is a complex left reduced ideal of $X$. For any $x \in X$, we have

$$
\operatorname{Re}\left(\mu_{A}(x)\right)=\operatorname{Re}\left(\mu_{A}(x * 0)\right) \geq \operatorname{Re}\left(\mu_{A}(0)\right)
$$

and

$$
\operatorname{Im}\left(\mu_{A}(x)\right)=\operatorname{Im}\left(\mu_{A}(x * 0)\right) \geq \operatorname{Im}\left(\mu_{A}(0)\right) .
$$

Since $x * x=0$ for all $x \in X$, the condition (3.4) implies that $\operatorname{Re}\left(\mu_{A}(x)\right) \leq$ $\operatorname{Re}\left(\mu_{A}(x * x)\right)=\operatorname{Re}\left(\mu_{A}(0)\right)$ and $\operatorname{Im}\left(\mu_{A}(x)\right) \leq \operatorname{Im}\left(\mu_{A}(x * x)\right)=\operatorname{Im}\left(\mu_{A}(0)\right)$. Therefore $\mu_{A}(0)=\mu_{A}(x)$ for all $x \in X$, that is, the complex valued membership function $\mu_{A}$ of $A$ is constant.

The proof of the following two theorems is the same as the proof of Theorems 3.11 and 3.12 .

TheOREM 3.15. Let $f: X \rightarrow Y$ be a homomorphism from $X$ to a $B C K / B C I$ algebra $Y$. If $B$ is a complex left (resp. right) reduced ideal of $Y$ with the complex valued membership function $\mu_{B}$, then the homomorphic preimage $f^{-1}(B)$ of $B$ under $f$ is a complex left (resp. right) reduced ideal of $X$ with the complex valued membership function $\mu_{f^{-1}(B)}$.

Theorem 3.16. Let $f$ be an endomorphism of $X$. If $A$ is a complex left (resp. right) reduced ideal of $X$ with the complex valued membership function $\mu_{A}$, then the complex fuzzy set $A[f]$ on $X$ with the complex valued membership function $\mu_{A[f]}$ defined by

$$
\mu_{A[f]}(x)=\mu_{A}(f(x))
$$

for all $x \in X$ is a complex left (resp. right) reduced ideal of $X$. 


\section{Conclusions}

Generally, the extension of crisp sets to fuzzy sets, in terms of membership functions, is mathematically comparable to the extension of $\mathbb{Z}$ (; the set of integers) to $\mathbb{R}$ (; the set of real numbers). That is, expanding the range of the membership function from $\{0,1\}$ to the unit interval $[0,1]$ is mathematically analogous to the extension of $\mathbb{Z}$ to $\mathbb{R}$. The development of the number set did not end with real numbers. Historically, the introduction of real numbers was followed by their extension to the set of complex numbers, $\mathbb{C}$. Hence, it may be suggested that a further development of fuzzy set theory should be based on this extension. In the context of set theory, the result of such an extension is the complex fuzzy set, i.e., a fuzzy set characterized by a complex-valued membership function. Based on such background, Ramot et al. introduced complex fuzzy set in their paper [1]. The complex fuzzy set is characterized by a membership function $\mu$ whose range is not limited to $[0,1]$ but extended to the unit circle in the complex plane. In this paper, we have used complex fuzzy sets to obtain the generalization of fuzzy set theory in BCK/BCI-algebras. We have introduced the notion of a complex subalgebra and a complex reduced left (right) ideal in a BCK/BCI-algebra, and have investigated related properties. We have provided characterizations of a complex subalgebra, and have discussed the homomorphic image (preimage) of a complex subalgebra and a complex left (right) reduced ideal. We will use the ideas and results of this paper to study various types of sub-structure in algebras in the future.

Acknowledgements. This research is supported by a grant of National Natural Science Foundation of China (11571281). The authors wish to thank the anonymous reviewer for his/her valuable suggestions.

\section{References}

[1] D. Ramot, R. Milo, M. Friedman and A. Kandel, Complex fuzzy sets, IEEE Transactions on Fuzzy Systems 10(2), (2002), pp. 171-186.

[2] D.E. Tamir and A. Kandel, Axiomatic theory of complex fuzzy logic and complex fuzzy classes, International Journal of Computers Communications \& Control, 6 (2011), no. 3, pp. 562-576. 
[3] Y. Al-Qudah and N. Hassan, Operations on complex multi-fuzzy sets, Journal of Intelligent and Fuzzy Systems 33 (2017), pp. 1527-1540. DOI:10.3233/JIFS-162428

[4] Y. S. Huang, BCI-algebra, Science Press, China (2006).

[5] J. Meng and Y. B. Jun, BCK-algebras, Kyungmoon Sa Co., Seoul (1994).

[6] A. Azam, B. Fisher and M. Khan, Common fixed point theorems in complex valued metric spaces, Numerical Functional Analysis and Optimization 32(3), (2011), pp. 243-253.

Department of Mathematics Education

Gyeongsang National University

Jinju 52828, Korea

e-mail: skywine@gmail.com

School of Mathematics

Northwest University

Xi'an 710127, China

e-mail: xlxin@nwu.edu.cn 